\title{
¿Un nuevo derecho para los niños y adolescentes?
}

Benjamín Aguilar Llanos

El ser humano en la primera etapa de su vida, es incapaz de valerse por sí mismo; es un ser en estado de necesidad natural; requiere de asistencia, protección, socorro para su sobrevivencia, pues de no ser así perecerá.

Este estado de insuficiencia, y la consecuente obligación de atenderlo ha dado lugar a que el Derecho regule una serie de instituciones jurídicas dirigidos directa o indirectamente a amparar al infante; tales como la Patria Potestad, Alimentos, Tutela, Patrimonio Familiar. Sin embargo, las particularidades propias del ser humano en su fase evolutiva desde que nace hasta salir a la capacidad, ha requerido una regulación propia; por ello se creó una legislación especial que se ocupará del infante; tal fue el Código de Menores de 1962.

Esta legislación especializada se dio en un contexto y dentro de una doctrina en la que el menor era un objeto de protección pues su debilidad debería motivar a la sociedad una compasión que desembocaría en un deber social para cubrir sus necesidades; este objeto del Derecho, indefenso y $\sin$ capacidad, fue regulado principalmente, en favor de los menores, cuyos padres, por razones diversas no estaban con él, y por ello se encontraban en una situación irregular; y en esa medida la sociedad lo enfrentó como uno de sus problemas por resolver.

Desde 1962 a la fecha, resulta un período prudente para preguntarnos si los principios que guiaron esa legislación fueron positivos y obtuvieron los resultados deseados, o más bien, fracasaron y por ello, tales principios tuvieron que ceder su lugar a otros, mucho más próximos a la dignidad del ser humano, que, no por un factor cronológico, deje de serlo o lo sea con una minusvalía.

El presente trabajo pretende descubrir si estamos frente a nuevas orientaciones en el trato a los infantes, y si estos nuevos principios son positivos para alcanzar los objetivos deseados. 


\section{Código de Menores de 1962. Sus orientaciones}

El 2 de mayo de 1962, se promulga la ley 13968, conocida como el Código de Menores; este Código se inspiró en la Declaración de los Derechos del Niño de la Organización de las Naciones Unidas; Código de Declaración de Oportunidades para el Niño del Octavo Congreso Panamericano del Niño de 1942, y la Carta de los Derechos de la Familia Peruana del Congreso de Protección a la Infancia de 1943.

Por mucho tiempo se dijo que este Código fue poco menos que notable, muy avanzado para su época y que debía servir de modelo para otros códigos del continente; y esta percepción positiva se derivaba de que este cuerpo de leyes consagraba la protección al binomio madreniño; una justicia especializada, una procuraduría, policía y patronato de menores, ordenaba establecer gabinetes de observación y orientación que deberían funcionar en las secciones preventivas de los establecimientos tutelares; el control de la madre gestante -hasta la etapa del puerperio- era obligación de los establecimientos de salud, los cuales, incluso, deberían de ser rodantes para hacer extensivo a mayor número de gestantes. Sin embargo, las bondades asistenciales de estas medidas, o no se aplicaron o fueron insuficientes para atender a una población infantil que superaba cualquier crecimiento razonable.

Por otro lado, si bien es cierto que se trató de insertar en un solo cuerpo de leyes todas las normas referidas a los infantes, ello no se consiguió en razón de la existencia de diversidad de normas dispersas, de diferente contenido como leyes laborales (2851), penales (Código Penal de 1924) entre otras. Sin embargo, la crítica severa que se hace de este Código está referida a que se concibe al menor como un objeto de tratamiento de programas de asistencia, y no como un sujeto de derecho (no hubo normas sobre opinión del menor, derechos de participación, capacidad jurídica relativa); no se trazaron políticas de Estado respecto a la población infantil, y ello por cuanto el Código estaba dirigido principalmente para el menor en situación irregular, dentro de los cuales se consideraba a los abandonados, los que se encontraban en peligro moral o estado peligroso, deficientes sensoriales y mentales, lisiados físicos y con necesidad temporal, y todos ellos constituían un problema para la sociedad, la cual debía establecer los medios para solucionarlos, y el Código de Menores principalmente, optó por la intitucionalización.

Es cierto que el Código contempló situaciones de menores, que sin estar en la condición de «irregulares», no se apartaban mucho de ello, en razón de que sus padres discutían la tenencia e incluso la patria po- 
testad exclusiva sobre ellos; y aquí debería intervenir el Juez, para dilucidar su situación, aplicando fríamente las normas que regían sobre el particular.

Al normar principalmente a los menores en situación irregular, este Código no previó la participación de la sociedad, ni la atención de una población infantil que requería de un compromiso de todos los ciudadanos para labrar un desarrollo óptimo de ellos.

\section{Contexto en el que se da el Código de los Niños y Adolescentes}

El Código de Menores de 1962 transcurrió sin producir cambios cualitativos en la población infantil; la atención a menores en situación irregular, que debió perseguir la reinserción familiar de estos menores, no fue cumplida. Es así que nace una imperiosa necesidad de promover una legislación más realista y acorde con los nuevos requerimientos, acentuados por el aumento de la población infantil. Los compromisos internacionales en favor de la infancia fueron consolidando la urgente necesidad de dar al país un nuevo texto sobre los infantes, el cual es dado el 24 de diciembre de 1992, a través del Decreto Ley 26102, denominado Código de los Niños y Adolescentes; código que entró en vigencia a los 180 días de su promulgación.

Ahora bien, para conocer los alcances, proyecciones, objetivos de este nuevo cuerpo de leyes, debemos referirnos al contexto en el que se da, lo que implica trabajar con algunos indicadores respecto a la infancia en el año de 1992; no sólo el estado de la infancia, sino las convenciones internacionales existentes a esa fecha, y que comprometieron al país a asumirlas como propias. Veamos:

\section{a) La Convención de los Derechos del Niño}

El 20 de noviembre de 1989 , en su 61 sesión plenaria, la Asamblea General de las Naciones Unidas aprueba la convención sobre los derechos del niño y abre a la firma, ratificación y adhesión de los países. No había transcurrido ni dos meses y medio de su aprobación, y el 26 de enero de 1990 la convención era firmada por 61 países. La convención reconoce la vulnerabilidad de los niños (estado de necesidad natural) y plantea sus derechos civiles, políticos, económicos, sociales y culturales como elementos de un conjunto de disposiciones interdependientes. 
Reconoce a los niños como sujetos con necesidades que evolucionan con la edad y la madurez, dándoles el derecho a participar en las decisiones que afectan tanto su presente como su futuro.

Esta convención reconoce cuatro grandes derechos de los niños:

- Derecho de supervivencia; esto es, niveles de vida adecuados y acceso a servicios médicos.

- Derecho al desarrollo; que incluye educación acceso a la información, al juego y el tiempo libre, a las actividades culturales y el derecho a la libertad de pensamiento, de conciencia y de religión.

- Derecho a la protección; cubre todas las formas de explotación y crueldad, separación arbitraria de la familia, y abusos en el sistema de justicia criminal.

- Derecho a la participación; libertad de expresar opiniones y poder manifestarse en cuestiones que afectan la propia vida.

El 3 de agosto de 1990, con resolución legislativa 25278 la Convención fue aprobada por el Congreso de la República y se convierte en el primer instrumento jurídico en el que se reconoce el principio trascendente del interés superior del niño, como norte y guía para cualquier decisión que afecte a la infancia. Al ratificarse la Convención sobre los derechos de los niños, estos derechos pasan a ser una responsabilidad del Estado y de la sociedad civil.

\section{b) Cumbre Mundial a favor de la Infancia}

El 29 y 30 de setiembre de 1990 se reunieron en Nueva York, cerca de 60 jefes de Estado y de Gobierno de todo el mundo y suscribieron una declaración mundial sobre la supervivencia, la protección y el desarrollo del niño, en la que se comprometieron solemnemente a atribuir alta prioridad a los derechos del niño, a su supervivencia, su protección y su desarrollo.

Se comprometieron a aplicar un programa de 10 puntos con el objeto de proteger los derechos de los niños y mejorar sus condiciones de vida:

- Ratificación y aplicación de la convención sobre los derechos del niño.

- Mejorar las condiciones de salud de los niños, fomentar la atención prenatal y reducir la mortalidad de niños menores de 5 años.

- Lograr un crecimiento y un desarrollo óptimo de los niños con medidas para erradicar el hambre y la desnutrición. 
- Fortalecer la función y la condición de la mujer; a fomentar la planificación responsable del tamaño de la familia.

- Respecto a la contribución de la familia el establecimiento de medidas para el cuidado de los niños.

- Establecer programas encaminados a reducir el analfabetismo y ofrecer oportunidades de educación a todos los niños.

- Mejorar la situación de los niños que viven en circunstancias especialmente dificiles.

- Establecer medidas para la protección del medio ambiente;

- Lucha contra la pobreza; lo que se reflejaría de inmediato en un mayor bienestar para los niños.

Esta cumbre mundial, solicita entre otras colaboraciones, la de los mismos niños para que participen en esta tarea.

\section{c) Estado de la infancia en el Perú al año de 1992}

La población del país se estimó en 22.5 millones.

Los menores de 15 años constituyeron el $37 \%$ y los comprendidos entre 15 y 64 años el $59 \%$ del la población.

Esperanza de vida al nacer : 65 años.

La tasa de mortalidad infantil era la tercera más alta de América Latina, después de Bolivia y Haití, y estaba estimada en 80 por mil nacidos vivos a 1990 .

La tasa de mortalidad materna se estimó en 303 por 100 mil nacidos vivos. La desnutrición crónica (talla/edad) en menores de 5 años afectó al 36.5\% en 1991, mientras que la desnutrición global (peso/edad) al $10.8 \%$.

La cobertura educativa a 1991 para niños de 0-2 y de 3-5 años alcanzó a $1.3 \%$ y $49.3 \%$ respectivamente, mientras que la cobertura para el grupo de edad 6-14 años era mayor de 90\%. Se estimó en $1.7 \%$ la tasa de analfabetismo masculino y en $17.4 \%$ la femenina (1991).

Una consecuencia adicional de la crisis se expresaba en la disminución del gasto social del Estado, verbigracia el sector Salud que disminuyó en $56 \%$ y $67 \%$ en 1981 y 1989 respectivamente.

El número de menores que trabajaba entre 6 y 14 años había aumentado de 376 mil en 1985 a 730 mil aproximadamente en 1991; $47 \%$ de los cuales eran niños. 


\section{d) Plan Nacional por la infancia de 1992-1995}

El 20 de noviembre de 1992 se expide el Decreto Supremo 090-92 PCM, por el cual se aprueba el plan nacional de acción por la infancia de corto plazo 1992 y mediano plazo 1992 - 1995; en el que se establece tres grandes objetivos relacionados con la supervivencia, la protección y el desarrollo de la infancia peruana:

- Objetivos de supervivencia

- Reducir la tasa de mortalidad infantil de 80 a 60 por mil nacidos vivos en 1995; reducir la mortalidad de menores de 5 años de 119 a 90 por mil nacidos vivos en 1995.

- Reducir la mortalidad materna de 303 a 220 por 100 mil nacidos vivos en 1995.

- Ampliar el acceso al agua segura y a la disposición adecuada de excretas.

- Objetivos de desarrollo

- Reducir los niveles de desnutrición grave y moderada en menores de 5 años.

- Universalizar la educación básica y primaria.

- Reducir la tasa de analfabetismo adulto.

- Objetivos de protección

- Mejorar la protección de los menores en ciscunstancias especialmente dificiles

\section{De la doctrina de situación irregular del menor a la doctrina de la atención integral al menor}

En la década del 80, casi todas las legislaciones latinoamericanas referidas a los infantes aún seguían la doctrina de la situación irregular del menor; y es en el Brasil con el Estatuto del Niño y Adolescente (Estatuto da crianca $e$ do adolescente) en donde el 13 de julio de 1990 se deja atrás la citada doctrina, para incorporar nuevos principios que ya habían sido recogidos en la Convención de los derechos del niño.

La doctrina de la situación irregular se caracteriza por lo siguiente:

a) Se considera al menor como un problema por vencer. 
b) El menor es objeto del derecho; su incapacidad natural, lo convierte en un ser digno de compasión.

c) Indistinción entre abandonados moral o materialmente, infractores de la ley, e incluso los de deficiencia física o mental.

d) Facultad discrecional de la magistratura especializada, para la declaración de abandono moral o material.

e) Legislación represiva.

f) Carencia de políticas sociales a favor de los infantes

En realidad situación irregular, es una forma o estado de vida del infante, y que llega a él por deficiencias condenables de la sociedad. No escudriñar cuales eran las causas, y atacar sólo los efectos, desembocó en una población infantil, que en gran número se vio condenada a no gozar de un desarrollo adecuado. Es pertinente dictar medidas para aquellos menores que requieren reeducación, pero también lo es el de legislar para obligar a los adultos para superar aquellas condiciones de vida que influyen negativamente en el infante.

A decir de Emilio García Méndez «la esencia de esta doctrina se resume en la creación de un marco jurídico que legitime una intervención estatal discrecional y omnipotente, sobre esta suerte de producto residual de la categoría infancia constituida por el mundo de los menores".

Los objetivos deseados por esta doctrina, como eran la protección y resocialización, han fracasado, y como muestra de ello debemos referirnos a los indicadores peruanos, en lo que atañe a los considerados niños de la calle, trabajadores infantiles, adolescentes infractores y demás.

Esta doctrina inspiró al Código de Menores de 1962, cuya normatividad estaba referida principalmente a esta categoría de infantes con limitadas, mínimas posibilidades de desarrollo, dadas las circunstancias adversas en que se encontraban.

Considerar al menor como problema, como objeto pasible de programas de asistencia por su incapacidad natural, condujo a la adopción de medidas principalmente de institucionalización de estos menores. Posteriormente se pudo comprobar que estas medidas fueron inoperantes, ineficaces y, por qué no decirlo, fracasaron.

Ahora bien, transitar de la doctrina de situación irregular a otra que comprenda su atención integral, supone incorporar nuevos principios, nuevas directrices, las cuales, por su misma gravitación podrían ir creando una nueva doctrina en torno al infante. Estos principios a incorporar podrían resumirse en los siguientes: niño sujeto, no objeto; interés superior del infante; considerar situaciones especiales que dificultan o impi- 
den su desarrollo; participación de la sociedad civil en el desarrollo del infante; conversión de las necesidades de los infantes en derechos y justicia especializada y, por qué no, abandonar el término menor.

Brevemente desarrollaremos estos conceptos.

\section{Principios de la doctrina de atención integral del infante}

\section{a) Niño sujeto, no objeto}

Por muchos años se consideró al niño como un ser que por su debilidad natural, era vulnerable, y al cual por ese estado de necesidad que atravesaba debía ser materia de protección, amparo, socorro. Se le trató como objeto de programas que el Estado debía llevar a cabo; no se pensó en él como persona que tiene derechos propios, y que según su desarrollo evolutivo podía ser considerado como agente de su propio desarrollo.

La incapacidad natural del infante, según la doctrina de la situación irregular, no le permitía participar como agente con derechos propios.

El niño y adolescente es un ser humano, y como tal con derechos fundamentales como los tienen los adultos. El niño evoluciona y en esta fase evolutiva, desarrolla sus capacidades cognoscitivas, su discernimiento se va acentuando: puede manifestarse, expresar sus opiniones. El infante es sujeto de derecho, y como tal hay que tomarlo en cuenta; esta capacidad de ser sujeto, implica tener derechos propios, no porque alguien se los haya concedido, sino que son inherentes a su condición de ser humano, y así la Convención de los Derechos del Niño y hoy el Código de los Niños y Adolescentes describe tales derechos que se pueden resumir en lo siguiente: supervivencia, desarrollo, protección y participación. En lugar de que el infante sea visto como un simple receptor o beneficiario de la asistencia social, debe ser concebido como un sujeto de derecho frente al Estado y la sociedad.

\section{b) Interés superior}

En el pasado, medidas que se referían a los menores, no fueron consideradas como centro de atención, en un orden prioritario, sino que, por el contrario, la infancia fue considerada como uno de los tantos problemas que debía superar la sociedad. La doctrina de situación irregular del menor, en la generalidad de los casos, tuvo una sola y casi exclusiva medida y ella fue la institucionalización (se masificó al niño, perdió su identidad, fue un anónimo); no hubo políticas claras para ayudar al in- 
fante (no sólo a los irregulares) a fin de que su etapa evolutiva le fuera favorable y posibilitar, posteriormente, su incorporación a la sociedad en condiciones positivas.

La doctrina de atención integral del niño y el adolescente considera como uno de sus más importantes principios el «interés superior», el cual implica que cualquier medida, acción, política que se dé en torno al niño y adolescente debe considerarse en lugar prioritario, lo que es más conveniente a él, lo que reporta como beneficio para su formación, lo que más le ayuda; en otras palabras, antes de considerar cualquicr interés, debe priorizarse, preferirse el interés del niño y el adolescente; su supervivencia, protección y desarrollo, debe estar por encima (superior) de todo; esto debe significar que cualquier política, acción normatividad, debe a la par ser favorable al niño y adolescente, no debe ser rígido, inflexible, pues más que las políticas y medidas está el niño y el adolescente, y que tales políticas, normas y acciones, no son un fin por sí mismos, sino medios que son útiles, en tanto y en cuanto, vayan en favor del infante. Este interés superior que debería tornar las normas que atañen al infante, como normas de orden público (de obligatorio cumplimiento), deben ser tenidas muy en cuenta por los creadores de políticas y normas, así como por los operadores del derecho y ejecutores de tales políticas.

El principio del interés superior establecido por la Convención y recogido por el Código de los Niños y Adolescentes, es una exigencia, una obligación y no una simple recomendación o llamado; es una orientación que conlleva un mandato para la actuación de las autoridades de gobierno y la familia.

\section{c) Niños y adolescentes en circunstancias especialmente dificiles}

La doctrina de la situación irregular del menor, agrupó en un solo tipo a una variedad de infantes con circunstancias adversas para su desarrollo; agrupó a los menores abandonados, en estado de peligro moral, en estado peligroso, deficientes sensoriales y mentales, lisiados físicos, con necesidad temporal, y para todos ellos dio una sola salida, la institucionalización. Sin embargo, obsérvese que estamos ante una variedad de situaciones, con sus propias particularidades y características y que en muchos casos debió motivar medidas administrativas o judiciales diferentes para cada caso. Ahora bien, no se trata de ignorar que existen niños o adolescentes que se encuentran en riesgo, o como lo llama la Convención en circunstancias especialmente dificiles, y que al hablar de circuns- 
tancias nos referimos a algo no natural, no inherente a los niños, a condiciones de vida que los pone en situación de debilidad o como llama UNICEF, vulnerabilidad que los impide desarrollarse.

Estos niños y adolescentes que se encuentran en circunstancias especialmente dificiles, son aquellos que viven con carencias afectivas, sociales, culturales, materiales y espirituales o por estar padeciendo de abusos, maltratos, explotación. A guisa de ejemplo podemos establecer hasta cinco categorías de niños o adolescentes en circunstancias especialmente dificiles:

- Niños y adolescentes en estrategias de sobrevivencia: los que realizan actividades de generación de ingresos.

- Víctimas de maltrato y abandono

- Niños o adolescentes de la calle

- Víctimas de la violencia armada

- Niños o adolescentes institucionalizados.

Obsérvese que estas circunstancias adversas pueden dar lugar a que los niños y adolescentes lleguen a cometer infracciones contra la ley, a los que en algún momento se les llamó menores de conducta antisocial y que incluso el Código de Menores de 1962, habló hasta de menores peligrosos; ahora bien, como es natural, cada una de estas circunstancias exige medidas especiales para modificarla a través de políticas adecuadas y coherentes para lograr el desarrollo de todos los infantes.

\section{d) Participación de la sociedad civil}

Considerar al menor en situación irregular y verlo como problema, como carga de la sociedad, implicó que las autoridades aborden el «problema» por la judicialización, esto es, la intervención del aparato judicial a fin de dictar medidas de "protección» que no significó otra cosa que recluir al menor. Además, la legislación se dirigió mayormente o quizás debemos decir exclusivamente, a regular la situación de aquellos menores con problemas; pero no se dijo algo respecto a la población infantil en general; no hubo normas referidas a la prevención, no se reguló en favor del niño en «situación regular»; a lo mejor por considerar que los deberes de atención a favor de ellos deberían circunscribirse exclusivamente al hogar, y no hacer participar al Estado, ni mucho menos a la sociedad, pues era un problema ajeno a ellos.

Una doctrina de atención integral, debe referirse a los niños y adolescentes en general, sin desconocer las diferentes condiciones en que 
puedan hallarse éstos; al respecto es ilustrativo lo que reconoce la Convención: "que aunque un niño esté adecuadamente nutrido (un derecho social), su derecho a desarrollarse plenamente no está protegido adecuadamente a menos que también se lo eduque (un derecho cultural y social) y proteja de ciertas cosas como la detención arbitraria (un derecho civil) y la explotación en el trabajo (un derecho socio-económico)».

Es en este contexto que el Código de los Niños y Adolescentes llama a participar a la sociedad, en una tarea que le es propia, y que se constituye como un deber de solidaridad social, y no como una gracia.

Ahora bien, la forma como participa la sociedad, esto es, los medios o caminos para integrarse al esfuerzo en el reconocimiento y ejercicio de los derechos de los niños es variado; así el Código ha creado el sistema nacional de atención integral del niño y adolescente, en el que deben participar no sólo el sector público, sino también los entes privados y la sociedad en general. Este sistema establece el Ente Rector, que dirige, planea, ejecuta, supervisa todos los planes y programas a favor de los niños y adolescentes, y en cuyo directorio, hay tres representantes de la sociedad civil, sin perjuicio de contar con los representantes de la Iglesia Católica.

La sociedad civil entera está comprometida con este esfuerzo, pues al hacerlo está luchando por su sobrevivencia y mejores condiciones de vida, en tanto que potenciar el desarrollo de la niñez, es labrar un futuro mejor para la misma sociedad.

\section{e) Conversión de necesidades en derechos}

Por muchos años se dijo que lo menores tenían una serie de necesidades y que los adultos debíamos de cubrir tales necesidades. Ahora bien, necesidad es carencia, ausencia, falta, privación, las mismas que requieren ser atendidas, pero quienes deben atender estas necesidades, por obvia razón, en primer lugar son los padres que han traído al mundo a estos seres, y cuando ellos faltan o ellos mismos se encuentran en carencia (que es el caso peruano), pues estas necesidades deberían ser atendidas por la sociedad. Sin embargo, ¿se puede exigir legalmente la satisfacción de estas necesidades?, diríamos que no, pues las necesidades se ubicarían dentro del plano del deber moral y como el incumplimiento del deber moral no acarrea sanción que no sea la moral, pues entonces estas necesidades se quedan como se quedaron insatisfechas por mucho tiempo.

Estas necesidades deberían trascender del plano moral (sin dejar lo moral) al plano de los derechos, lo cual se logró al dejar atrás la doctrina de la situación irregular; ello ha significado que el deber moral que 
tenía la sociedad para con su población infantil, se convierta en deber civil, y por lo tanto en capacidad de demandarse su cumplimiento.

El niño y adolescente dejó de ser considerado como objeto, y a su condición de sujeto exhibe una serie de derechos propios e inherentes a su calidad de ser humano, y en tal mérito, en aptitud de que a sus derechos les corresponda la acción para demandar su cumplimiento.

Todo ello significa que ya no se suplique o implore para que se satisfagan las necesidades de los infantes, sino que se demande coercitivamente se cumplan las obligaciones de atención de los infantes, esto es reconocer y ejecutar sus derechos.

\section{f) Justicia especializada}

Se ha mencionado las diversas situaciones en las que se puede encontrar el niño y adolescente; situaciones o circunstancias que le pueden ser adversas para su desarrollo, o peor aún que le conduzcan a entrar en conflicto con las normas de convivencia social; en esa circunstancia, atender el caso de estos infante, requerirá de conocimientos especiales.

El problema así presentado no sólo es legal, debe tratarse como problema humano, y por ello requerirá de una magistratura especializada. Es cierto que la legislación anterior ideó la magistratura especializada pero no la implementó, como si viene ocurriendo en el presente con los juzgados de Familia y Tribunal de Familia; magistratura que no actúa sola ( al menos no debería serlo) sino que está acompañando de un equipo multidisciplinario de profesionales, y que atenderán el caso del infante, no sólo desde el ángulo de aquel que se puso al margen de la ley, sino que verá su entorno familiar, las causas que llevaron al infante a esa situación, su nivel educativo y demás; ello a no dudar, contribuirá a un mejor trato al infante posibilitando su reinserción social.

Para concluir con este punto 4 , debemos referirnos mas que a un principio a algo que sin serlo resulta conveniente, como es abandonar el término menor. La palabra menor proviene de la voz latina «minon»; se es menor en comparación con la persona que ya es mayor.

La normatividad jurídica precedente al Código de los Niños y Adolescentes, al referirse al infante, lo denominó menor, y ello en atención a su edad cronológica inferior a los 18 años (antes 21 ), en la que sale a la capacidad; la minoría de edad, entonces representó las primeras etapas evolutivas de desarrollo del ser humano, diferenciándose con aquellos sujetos que ya alcanzaron su plenitud.

Ahora bien, el que el infante, menor, esté en proceso de formación, no lo hace menos que los mayores, pues es un ser racional y libre (o 
potencialmente libre), con derechos propios relativos a su especialidad. Sin embargo, se consideró al menor no sólo en contraposición al mayor por la edad, sino que la minoridad vino a ser entendida como una suerte de minusvalía, un ser disminuido, y por sus carencias naturales, digno de compasión y por ello objeto de medidas de asistencia.

Entonces la minoridad no solo significó una circunstancia en razón de no haber alcanzado una determinada edad, sino un ser menor a aquellos que habían llegado a la mayoridad; y ese "menor" significó restar posibilidades y despriorizar su atención.

La Convención de los derechos del niño ya no se refiere al infante como menor, sino que lo denomina niño, el cual se es desde la concepción hasta los 18 años (respetando legislaciones que fijen edades menores para salir a la capacidad). El Código de los Niños y Adolescentes hace una mayor precisión y diferencia al niño del adolescente; el primero lo es desde su concepción hasta los 12 años, y el adolescente desde los 12 cumplidos hasta llegar a los 18 años.

El haber abandonado el término menor no sólo significa un cambio de términos sino que conlleva toda una connotación jurídica, pues la ninez y la adolescencia son etapas de la evolución del ser humano y que no deben significar disminución, resta de derechos, o peor aún, minusvalía, pues se trata del sujeto de derechos que atraviesa un desarrollo natural y que por ello no lo hace menos que nadie.

\section{Aportes del Código de los Niños y Adolescentes}

\section{a) Políticas de atención}

Dentro de las nuevas orientaciones que se imprimen a la legislación de los infantes encontramos la doctrina de la atención integral del niño y adolescente, correspondiéndole a la sociedad una responsabilidad gravitante. La necesidad de diseñar políticas a favor de los infantes se hace indispensable, políticas que deben ser Estado y no sólo de gobiernos, pues de por medio está el futuro de la sociedad, representada por su población infantil.

En el contexto y alejándose de lo que fue la orientación del Código de menores, el actual Código de los Niños y Adolescentes, en su libro segundo, al referirse al Sistema Nacional de Atención Integral al Niño y Adolescente, establece que las políticas de atención estarán orientadas a desarrollar: 

cuada.

- Programas de prevención que garanticen condiciones de vida ade-

- Programas de promoción, que motiven su participación y de su familia y que permitan desarrollar sus potencialidades.

- Programas de protección que aseguren la atención oportuna cuando enfrenten situaciones de riesgo.

- Programas de asistencia para atender las necesidades cuando se encuentren en circunstancias especialmente dificiles, y

- Programas de rehabilitación que permitan la recuperación física y mental y que le ofrezcan atención especializada.

Queda claro que en esta tarea están comprometidos no sólo los gobiernos de turno, sino la sociedad entera, la misma que vía representantes, está formado parte del Ente Rector del Sistema de Atención Integral del Niño y Adolescente. Estas políticas, en resumen, deben dirigirse a asegurar la supervivencia, desarrollo, protección y participación del niño y adolescente y deben enmarcarse dentro de los nuevos principios que inspiran la doctrina de los infantes, esto es, ser considerado el niño y adolescente como sujeto de derechos, y su interés superior, que será desarrollado más adelante.

En la actualidad, estas políticas están siendo plasmadas en los planes de atención del niño y adolescente. El primero de ellos comprendió el año 92, y el segundo del 93 al 95, con metas que en algunos casos se cumplieron, como es el caso de la reducción de la mortalidad infantil, y en otros, no se pudo llegar siquiera a aproximarnos a la meta, como por ejemplo la mortalidad materna y la desnutrición crónica, entre otros. En el presente (octubre de 1996) se culmina la elaboración del plan nacional de atención integral del niño y adolescente del 96 al 2000 con metas más ambiciosas, y con un compromiso de la sociedad para su población infantil.

El sistema de atención integral al niño y al adolescente tiene la finalidad de orientar, integrar, estructurar, coordinar, supervisar y evaluar las políticas, planes, programas y acciones a nivel nacional destinados a la atención integral de niños y adolescentes.

Como ha quedado expresado, una tarea tan trascendente no puede quedar sólo como responsabilidad del gobierno, por ello integran el sistema, aparte de los órganos sectoriales e instituciones públicas, gobiernos regionales, locales e instancias descentralizadas de gobierno que prestan servicios de atención al niño y adolescente, también las instituciones privadas y las organizaciones comunales y sociales de base que lleven a cabo programas a favor de los niños y adolescentes. 
Por Ley 26518, modificada luego por Ley 26621 se establece que es el Ente Rector el órgano central del sistema, con autonomía técni$\mathrm{ca}$, funcional, administrativa y es la encargada de dirigir y formular las políticas, planes y programas sectoriales e institucionales de atención al niño y al adolescente.

El Ente Rector tiene un directorio cuyos integrantes no sólo pertenecen a la administración pública (ministerios de la Presidencia, Salud, Educación, Justicia, Trabajo y Promoción Social, Economía) sino también por representantes de la Iglesia Católica, y tres representantes de la sociedad. Ahora bien, en el momento no hay todavía elección de los representantes de la sociedad civil, sin embargo, aspiramos a que pronto puedan ser designados ahora que el reglamento de la Ley 26518 ya está en vigencia.

Creemos importante que estén presentes en el Directorio del Ente Rector, como representantes de la sociedad, los clubes de madres, vaso de leche, los empresarios (Confiep), los colegios profesionales, y otros; lo que indudablemente enriquecería el debate y las decisiones dentro del Ente Rector, para trazar políticas realistas e inteligentes en beneficio de nuestra población infantil.

\section{a) Interés superior del niño y adolescente}

La convención de los derechos del niño incorpora este principio por primera vez en un texto internacional, sobre el cual debe reposar cualquier política sobre infantes. Este interés superior implica poner en primer plano al niño y adolescente, cuando se delinien políticas, programas, acciones priorizando su atención.

El interés superior referido al infante y que debe contestar a la pregunta ¿qué es lo que más conviene al niño o adolescente? Debe guiar las políticas de su atención. Este interés superior lo referimos al plano político social, cultural y legal.

\section{b.1) Interés superior en lo político}

El desarrollo pleno del niño y adolescente debe estar siempre y en primer lugar en la agenda de los políticos, de los que tienen poder de decisión en el país. Debe ser puesto en primer lugar, en cualquier política de gobierno no sólo como un acto de socorro para una población a la que suele llamarse vulnerable, sino como una medida inteligente de inversión social; pues el desarrollo integral de los niños y adolescentes es el desarrollo de una sociedad en el futuro. 
Suele despriorizarse la atención de los infantes, cuando los países se encuentran en serias dificultades económicas. Aun en esas circunstancias creemos que ello no debería acontecer, que en esas situaciones, deben trazarse y cumplirse políticas sociales, dentro de las cuales y en primer lugar debe estar la atención de la población infantil.

\section{b.2) Interés superior en lo social}

Aquí utilizamos el término social para referirnos a la sociedad; y es así que entendemos, que no sólo los políticos, los hombres de gobierno deben atender la necesidades de los infantes; sino que este deber, es de la sociedad en pleno; el sector privado, los empresarios, trabajadores, organizaciones sociales de base deben plantearse como reto prioritario, acciones a favor de los infantes.

$\mathrm{La}$ contribución de ellos como un deber de solidaridad social es necesario y urgente para construir una sociedad fuerte, que les permita avizorar el futuro, como algo deseable y no como una pesadilla por venir.

\section{b.3) Interés superior en lo cultural}

Conocido es que, cuando una sociedad desea superar sus limitaciones y deficiencias, debe invertir en su población infantil, y la inversión más segura está dada a través de la educación, posibilitando con ello generaciones pensantes y en mejores condiciones para afrontar los retos del futuro, tal como ha ocurrido con las sociedades desarrolladas, que para llegar a serlo, han invertido en la educación de su pucblo. Entonces, priorizar la educación no sólo es cumplir con las necesidades de los infantes, sino que ello resulta urgente para la misma sobrevivencia de la sociedad; priorizar la educación de los infantes es atender el interés superior de él; la educación es parte esencial y primordial de la atención integral del infante.

\section{b.4) Interés superior en lo legal}

El Perú, al formular la legislación referida a los infantes, acorde con la Convención de los derechos del niño, incorpora el interés superior como principio, y así el Código de los Niños y Adolescentes, en su artículo VII del Título Preliminar refiere «En toda medida concerniente al niño y adolescente que adopte el Estado a través de los poderes Ejecutivo, Legislativo y Judicial, Ministerio Público, gobiernos regionales, 
gobiernos locales y sus demás instituciones, así como en la acción de la sociedad, se considerará el interés superior del niño y adolescente y el respeto a sus derechos».

En la exposición de motivos del Código, se señala que este principio tiene su sustento en el respeto a la dignidad de la persona humana reconocida en los artículos l y 3 de la Constitución Política del Perú. Se señala que el derecho subjetivo lo concibe como aquel que se sustenta en la dignidad misma del ser humano, en tal sentido los derechos subjetivos no serán «numerus clausus» limitados por la norma, sino que tutelarán todos los intereses existenciales del ser humano. En tal mérito, toda norma referida al infante debe interpretarse por lo que más convenga al niño o adolescente; más aún, creemos que si existiera un conflicto entre la norma y los intereses de un infante, que supuestamente la norma debe cautelar, deberíamos preferir el interés directo e inmediato del infante; esto debe ser bien entendido sobre todo por los operadores del derecho; aquellos que tengan poder de decisión sobre situaciones en que se ventilen intereses de infantes.

La legislación debe propender a una paz social; y la legislación especializada del infante debe buscar la atención óptima del infante; por lo tanto el sistema jurídico positivo debe estar orientado a ello. Ahora bien, las diversas y variadas situaciones en que se encuentran los infantes rebasa en muchos casos a la normatividad, o quizás nos lleva a un conflicto con la ley positiva. Si ello se diera, no debemos preferir la norma, o ante ausencia o vacío de norma (laguna del derecho) debemos estar a lo que objetiva y realistamente convenga más al infante, según su particular situación. Aquí el proverbio latino "dura lex sex lex« no debe funcionar, pues más que la ley, nos debe preocupar el interés del infante.

En conclusión, el principio del interés superior, debe tenerse como un criterio orientado para resolver conflictos de derechos en que puedan verse involucrados los infantes.

\section{c) Atención integral del niño y adolescente}

El infante por el período evolutivo en el que está insertado, requiere de atenciones que cubran sus múltiples y variadas necesidades (derechos). Estas necesidades se ubican en el plano de su supervivencia (salud, alimentación); desarrollo (educación, recreo); protección (contra el abuso y explotación) y participación (derecho de opinión). Estas necesidades convertidas en derechos posibilitarán que el infante se incorpore en condiciones favorables a la sociedad.

Pues bien, propender a satisfacer estas necesidades, significa atender a 
su desarrollo integral y en ese sentido, las políticas, planes y programas referidas al infante, deben orientarse a lograr este objetivo trascendente.

La legislación anterior, inspirada en la doctrina de la situación irregular del menor, redujo, sesgó la atención del infante, circunscribiéndola en la mayoría de los casos, a aquellos menores con ciertas deficiencias que imposibilitaban su desarrollo, e incluso considerándolos como problema social. Esta doctrina no previó una atención integral a la población infantil; es cierto que el Código de menores de 1962 previó algunas circunstancias distintas a los menores en situación irregular, y lo hizo al referirse a los matrimonios desavenidos, en donde los padres discutían la tenencia de los hijos - problema que afecta a un sector minoritario y casi siempre pudiente de la población- pero no reguló a favor del grueso de la población infantil. Temas como supervivencia, desarrollo, participación quedaron al margen del Código.

El niño y adolescente es una persona humana, sujeta de derechos, que al atravesar la primera etapa de su vida, se encuentra con carencias naturales, que en la generalidad de los casos no puede atender por sí mismo, y por ello se hace indispensable que sus progenitores (en primer lugar) atiendan estos requerimientos. Pero muchas veces los padres no pueden hacerlo, por sus propias carencias, entonces estas necesidades transcienden el ámbito hogareño y se convierten en necesidades sociales, que es necesario y urgente atender. La sociedad debe propiciar las circunstancias especiales para que el niño y adolescente pueda desarrollarse y convertirse mañana más tarde en sujeto idóneo y apto para su familia y la sociedad entera.

Se ha señalado que el niño y adolescente tiene diversidad de necesidades, por ello su atención debe ser cubierta tratando de comprender todas estas necesidades, y en este sentido hace bien el Código de los Niños y Adolescentes, cuando en el artículo 32, establece políticas generales de atención, tratando de cubrir las diversas situaciones en que se encuentra la población infantil, y por ello consigna programas de prevención, promoción de protección (para situaciones de riesgo) y de rehabilitación.

\section{d) La costumbre como fuente de derecho}

Se ha señalado que el Perú es un país pluricultural, un país a la vez moderno y antiguo; en donde impera el derecho occidental, pero que también en mucho lugares, impera sus usos y costumbres, lugares remotos en donde la legislación positiva, o se conoce poco o no se conoce. 
Reconocer esta situación heterodoxa del país, es actuar realistamente; por cuanto nuestras comunidades nativas y selváticas se rigen por su propia ley, que es una costumbre inveterada, y que su continuidad, su publicidad y aceptación, la hacen obligatoria.

Por todo ello, creemos acertado que el Código de los Niños y Adolescentes recoja aunque en forma supletoria, la costumbre como fuente de derecho. Por defecto o deficiencia de la ley se aplican los usos y costumbres; así reza la última parte del artículo VI del Título Preliminar del Código de los Niños y Adolescentes.

La ley regula una situación de hecho, pero estos hechos son cambiantes, cambios que superan a la ley. Esto es, la realidad termina superando a la ley, con lo cual la ley ya no alcanza a cubrir ese fenómeno o lo cubre parcialmente, y en este caso viene en auxilio los usos y costumbres inveterados, que no siendo normas escritas, pero por su amplitud y por ser producto de las situaciones que se repiten en un lugar determinado, tienen una mayor cobertura de lo que podría tener la ley.

En este contexto resulta interesante que se haya recogido supletoriamente la costumbre como fuente de derecho para los infantes; usos y costumbres que tienen que ir muy de la mano con el interés superior del niño y adolescente y que incluso podría dar lugar a no aplicar una determinada norma si esta, en un caso particular y especial, no conviene al infante, y los usos y costumbres de ese lugar aconsejan no aplicar la citada norma, por no ser lo más conveniente a los intereses del niño y adolescente.

Conocemos de lugares muy apartados en los cuales el fenómeno de la violencia ha dejado una población infantil huérfana; si en estos casos tuviéramos que guiarnos por la normatividad legal sobre tutela (discernimiento, garantía, inventario para la tutela, artículo 520 del Código Civil) para que alguien se encargue de cuidar al infante con el título de Tutor, tendríamos que concluir que el huérfano se quedaría sin Tutor. En estos lugares el concepto de familia que impera no es la nuclear (padres - hijos), sino la extensa (padres-hijos y otros parientes), y por ello los parientes más lejanos tienen la obligación que les impone la comunidad de cuidar de estos infantes desvalidos, obligación que se hace extensiva incluso a los parientes espirituales (padrinos). Aquí tenemos un ejemplo de cómo los usos y costumbres, incluso superan a la norma legal.

\section{A manera de conclusión}

Numerosas convenciones, foros, conversatorios, seminarios se desarro- 
llan en torno al infante; a la par que existen un número importante de textos nacionales e internacionales sobre el niño y adolescente; todo ello sin perjuicio del compromiso asumido por más de 60 gobernantes y jefes de gobierno, de priorizar la atención de sus poblaciones infantiles. Todo esto está revelando que estamos en un tiempo de urgencia y felizmente así lo han entendido los responsables de las decisiones de un país.

Se criticó mucho que no se priorizara la atención de los infantes, se arguyó con mucha razón, que al no merituar réditos políticos se desatendió el desarrollo de la población infantil, con lo cual a la sociedad misma se ponía en peligro, pues no invertir o no hacerlo a tiempo en la generación del futuro, era condenarse a sí misma. Parece ser que nos hemos dado cuenta, ojalá sea a tiempo, de que se hace indispensable que el niño y adolescente, debe ser la atención prioritaria de una sociedad que se requiere proclamar justa. Los planes de gobierno, los compromisos sociales deben pasar necesariamente por atender los requerimientos de los infantes, considerándolos como sujetos de derechos.

El niño y adolescente debe ser la razón de unión de los miembros de una sociedad; debe convocarnos para una tarea que nos comprometa a todos, tanto a los adultos como a los mismos niños y adolescentes.

Resulta grato referirnos a planes de corto y mediano plazo para atender a los niños y adolescentes, y que en dichos planes no sólo se encuentren comprendidos como responsables el sector público, sino la sociedad entera, a través de sus trabajadores, empresarios, profesionales, organizaciones sociales de base, iglesia; pues la tarea es de todos, porque a todos nos interesa y porque todos saldremos beneficiados si priorizamos a la atención del niño y adolescente.

Sc ha desarrollado en el presente artículo, los principios que informan la nueva doctrina de la atención integral del niño y adolescente, el cual como sujeto de derecho debe participar según sus posibilidades en su desarrollo; que sus necesidades son derechos y deberes para la sociedad; que su atención no debe ser sesgada sino integral; que cualquier política, programa, medida, acción en torno al infante debe guiarse prioritariamente por lo que más convenga a sus intereses. Y todo ello por cuanto, como ser humano que es, sujeto de derechos como cualquier adulto, pero con particularidades propias de su desarrollo evolutivo, que lo hacen merecedor de medidas de protección. Es en este contexto que podemos establecer que los derechos fundamentales consagrados en nuestra Constitución y que explican la teoría de los derechos humanos, debe ser una política que en particular deberá referirse a los niños y adolescentes, pues los primeros derechos humanos son de ellos 
por su particular situación de indefensión, requerimientos naturales que los hacen vulnerables y por ello debemos todos, la sociedad en pleno, proteger sus derechos humanos.

\section{Bibliografía Consultada}

Baralta, Alessandro. "La niñez como arqueología del futuro", en $E l$ 1995 Derecho y los chicos. Bianchi, María (comp.). Buenos Aires. Espacio Editorial.

García Méndez, Emilio. Derecho de la Infancia - Adolescencia en Amé1994 rica Latina. De la situación irregular a la protección integral. Santa Fe de Bogotá. Fórum. París.

Pizarro, Crisóstomo. Niñez y Democracia. Bogotá. Unicef. 1995

García Méndez, E. La Convención internacional de los Derechos del niño 1995 y las politicas públicas. Documento de trabajo oficina regional de Unicef para América Latina y el Caribe. Santa Fe de Bogotá.

Mendizábal, Oses. Derecho de Menores. Tcoría General (España).

Al.arcón, Walter. Entre calles y plazas. DEC - ATC, IEP.

Verdera, Francisco. El trabajo infantil en el Perú IEP.

Ministerio de Justicia. Los Derechos del Niño en el Perú Actual. Unicef. 1991

Ley Federal Brasileña 8.069/90. Estatuto Da Crianca e do Adolescente. 